\title{
Relaxation Dynamics of Semiflexible Polymers
}

\author{
Y. Bohbot-Raviv, ${ }^{1}$ W. Z. Zhao, ${ }^{2}$ M. Feingold, ${ }^{2}$ C. H. Wiggins, ${ }^{3,5}$ and R. Granek ${ }^{4,5, *}$ \\ ${ }^{1}$ Department of Materials and Interfaces, Weizmann Institute of Science, Rehovot 76100, Israel \\ ${ }^{2}$ Department of Physics and The Ilse Katz Center for Nanotechnology, Ben-Gurion University, Beer Sheva 84105, Israel \\ ${ }^{3}$ Department of Applied Physics and Applied Mathematics and Center for Computational Biology and Bioinformatics, \\ Columbia University, 500 West 120th Street, New York, New York 10027, USA \\ ${ }^{4}$ Department of Biotechnology Engineering and The Institute for Applied Biosciences, Ben-Gurion University, \\ Beer Sheva 84105, Israel \\ ${ }^{5}$ The Kavli Institute for Theoretical Physics, University of California, Santa Barbara, California 93106, USA
}

(Received 2 July 2003; published 3 March 2004)

\begin{abstract}
We study the relaxation dynamics of a semiflexible chain by introducing a time-dependent tension. The chain has one of its ends attached to a large bead, and the other end is fixed. We focus on the initial relaxation of the chain that is initially strongly stretched. Using a tension that is self-consistently determined, we obtain the evolution of the end-to-end distance with no free parameters. Our results are in good agreement with single molecule experiments on double stranded DNA.
\end{abstract}

Semiflexible polymers, such as DNA, $f$-actin, and microtubule filaments, are common examples of biomaterials. The near equilibrium dynamics of semiflexible polymers in solutions have been extensively studied experimentally [1] and theoretically [2-4] in the past decade, both because of the possible implications in biology and because their dynamics is rather different from the flexible chain dynamics.

The far from equilibrium dynamics of such systems received much less attention. Perkins et al. [5] studied the shape of relaxing DNA that is hydrodynamically stretched. Their results were interpreted by Brochard and co-workers [6], who considered the propagation along the chain of the relaxing tension. However, their work builds on the freely jointed chain model and so does not include bending energy. Therefore, it may not be applied to strongly stretched chains even when their length $L$ is much longer than their persistence length $L_{p}$. The strong stretching regime corresponds to tensions $\sigma \gtrsim k_{B} T / L_{p}$ associated with extensions above $0.5 \mathrm{~L}$. For such tensions, excess length-associated with bending energy dominated undulations - is being pulled out.

Modern experiments on single DNA molecules often use a bead that is attached to one or both ends of the chain. This allows one to exert a controlled force on the chain by means of optical or magnetic traps [7-10]. When the end is released, the contraction of the chain involves motion of the bead with a time-dependent velocity. The Stokes force acting on the bead, which is transmitted to the DNA as tension, slows down the dynamics [10]. Hence, the interpretation of such experiments requires a detailed theory that describes the dynamics of the DNA-bead complex. The purpose of this Letter is to provide such a description. We introduce a time-dependent tension that is used to calculate the end-to-end distance. We solve for this tension by relating it to the Stokes drag acting on the bead, which, in turn, is a result of the DNA contraction.
This allows us to fully describe the short time evolution of the end-to-end distance.

A time-dependent tension has been previously introduced $[11,12]$ and, in particular, has been used to describe the propagation of equilibrium tension fluctuations [3]. A key feature in these studies is that the propagation of polymer density (or, equivalently, tension) is given as a ratio of the compression modulus $B$ and a longitudinal drag coefficient. The compression modulus is found to be large and depends on the local tension as $B \sim \sigma^{3 / 2}[12]$, which leads to a tension propagation time $\tau_{\ell}$ shorter than the transverse undulation relaxation time by a factor of $4\left(k_{B} T / L_{p} \sigma\right)^{1 / 2}$ [12]. For the large tensions considered here, this factor is larger than 10. This implies that tension can be assumed uniform along the chain when we describe the dynamics of transverse undulations. Therefore, the propagation of the tension and the associated longitudinal drag coefficient become irrelevant. Only the slow variables, i.e., the transverse undulations and the bead velocity, limit the motion. The uniform tension assumption is also justified considering the time resolution of our experiment. The tension propagation time is [12] $\tau_{\ell} \simeq$ $0.04\left(k_{B} T / L_{p} \sigma\right)^{1 / 2} \eta L^{2} / \sigma$, where $\eta$ is the solvent viscosity. For the tensions and chain lengths that we use in experiment, $\sigma \simeq 10 k_{B} T / L_{p}$ and $L \simeq 20 \mu \mathrm{m}$, this leads to $\tau_{\ell} \simeq 0.006 \mathrm{~s}$, significantly shorter than the experimental time resolution, $0.04 \mathrm{~s}$.

The bending energy $\mathcal{H}_{o}$ of a semiflexible polymer is well described by the wormlike chain (WLC) or KratkyPorod model $[2,3,8]$. When an external force, $\sigma$, acts at the polymer ends pulling them apart, the energy becomes $\mathcal{H}=\mathcal{H}_{o}-\sigma z$, where $z$ is the end-to-end distance of the chain. In this model, the chain conformation energy is

$$
\mathcal{H}=\frac{\kappa}{2} \int_{0}^{L} d s\left(\frac{\partial^{2} \mathbf{R}}{\partial s^{2}}\right)^{2}-\sigma z,
$$


where $\mathbf{R}(s)$ is the 3D position vector of the $s$ polymer segment, $\kappa$ is the bending constant, and $L$ is the total arclength of the chain. The term $\left(\partial^{2} \mathbf{R}\right) /\left(\partial s^{2}\right)$ evaluates the curvature at point $s$. When $\sigma=0$, this energy leads to a persistence length $L_{p}=\kappa / k_{B} T$, that describes the arclength over which the local tangents decorrelate. The equilibrium force-extension relation, which results from the WLC model, has been investigated by Marko and Siggia [8] and verified in a wide range of experiments $[7,8]$.

We now consider the dynamics of a chain-bead system. The system we study consists of a large bead attached to an end of a semiflexible polymer, while its other end is fixed. Although the ratio $L / L_{p}$ can take any value, for $L \gtrsim L_{p}$ our treatment below applies only to the strong stretching regime, where roughness is small. The WLC Hamiltonian adopted for this case includes a timedependent tension $\sigma(t)$ that replaces the constant tension $\sigma$ [11]. $\sigma(t)$ will be identified as the Stokes drag acting on the bead, which is transmitted to the polymer itself as an external force. It acts as a time-dependent Lagrange multiplier conjugated to the extension $z(t)$.

We denote by $\mathbf{R}(s, t)$ the position of a polymer segment $s$ at time $t$, and by $\mathbf{h}(s, t)$ the $2 \mathrm{D}$ component of $\mathbf{R}(s, t)$ perpendicular to the end-to-end vector, $\mathbf{h}=\left[h^{(\alpha)}, h^{(\beta)}\right]$. The extension $z(t)$, in the small roughness approximation, is given by

$$
z(t) \simeq \int_{0}^{L} d s\left[1-\frac{1}{2}\left(\frac{\partial \mathbf{h}(s, t)}{\partial s}\right)^{2}\right] .
$$

In this approximation, the Hamiltonian of Eq. (1) becomes

$$
\mathcal{H}(t)=\frac{1}{2} \int_{0}^{L} d s\left[\kappa\left(\frac{\partial^{2} \mathbf{h}}{\partial s^{2}}\right)^{2}+\sigma(t)\left(\frac{\partial \mathbf{h}}{\partial s}\right)^{2}\right],
$$

where the constant $-\sigma(t) L$ has been omitted.

The stochastic motion of the polymer is described by the Langevin equation for $\mathbf{h}(s, t)$ [4]

$$
\begin{aligned}
\frac{\partial \mathbf{h}(s, t)}{\partial t}= & \int_{0}^{L} d s^{\prime} \Lambda\left(\left|s-s^{\prime}\right|\right) \\
& \times\left[-\kappa\left(\frac{\partial^{4} \mathbf{h}}{\partial s^{\prime 4}}\right)+\sigma(t)\left(\frac{\partial^{2} \mathbf{h}}{\partial s^{\prime 2}}\right)\right]+\mathbf{f}(s, t) .
\end{aligned}
$$

Here $\Lambda(s)=1 / 8 \pi \eta s$ is the Oseen hydrodynamic interaction kernel obtained from the diagonal terms of the Oseen tensor and $\mathbf{f}(s, t)$ is thermal white noise. Since the tension $\sigma(t)$ originates from the Stokes friction force acting on the bead, it may be related to the dynamics of the extension $\langle z(t)\rangle$, namely,

$$
\sigma(t)=-\zeta \frac{\partial\langle z\rangle}{\partial t},
$$

where $\zeta=6 \pi \eta b$ is the friction constant of the bead of radius $b$. In Fourier space, Eq. (4) becomes

$$
\frac{\partial \mathbf{h}_{k}(t)}{\partial t}=-\omega_{k}(t) \mathbf{h}_{k}(t)+\mathbf{f}_{k}(t),
$$

where $\quad \omega_{k}(t)=\Lambda_{k}\left[\kappa k^{4}+\sigma(t) k^{2}\right]$ and $\Lambda_{k} \simeq$ $\ln (1 / k a) / 4 \pi \eta$. Here $a$ is a short length scale cutoff chosen as the thickness of the polymer. The correlation function of $\mathbf{f}_{k}(t)$ is given by the fluctuation-dissipation theorem

$$
\left\langle f_{k}^{(\alpha)}(t) f_{-k}^{(\beta)}\left(t^{\prime}\right)\right\rangle=2 k_{B} T L \Lambda_{k} \delta_{\alpha \beta} \delta\left(t-t^{\prime}\right),
$$

where $\alpha$ and $\beta$ denote the two transverse directions.

Solving the Langevin Eq. (6) allows us to calculate the equal time undulation correlation function in Fourier space

$$
\begin{aligned}
\left\langle\mathbf{h}_{k}(t) \cdot \mathbf{h}_{-k}(t)\right\rangle= & \left\langle\mathbf{h}_{k} \cdot \mathbf{h}_{-k}\right\rangle_{o} e^{-2 \Omega_{k}(t)} \\
& +4 k_{B} T L \Lambda_{k} \int_{0}^{t} d t^{\prime} e^{-2\left[\Omega_{k}(t)-\Omega_{k}\left(t^{\prime}\right)\right]},
\end{aligned}
$$

where $\Omega_{k}(t)=\int_{0}^{t} d t^{\prime} \omega_{k}\left(t^{\prime}\right)$ and $\left\langle\mathbf{h}_{k} \cdot \mathbf{h}_{-k}\right\rangle_{o}$ is the initial undulation correlator. Since the polymer is assumed to be initially in equilibrium, we take

$$
\left\langle\mathbf{h}_{k} \cdot \mathbf{h}_{-k}\right\rangle_{o}=\frac{2 k_{B} T L}{\sigma_{o} k^{2}+\kappa k^{4}},
$$

where $\sigma_{o}$ is the initial tension, i.e., the pulling force on the bead prior to its release. Accordingly, the mean extension after the release, in the small roughness approximation, is

$$
\frac{\langle z(t)\rangle}{L}=1-\frac{1}{2 L^{2}} \sum_{k=\pi / L}^{\pi / a} k^{2}\left\langle\mathbf{h}_{k}(t) \cdot \mathbf{h}_{-k}(t)\right\rangle .
$$

The corresponding initial extension is $\langle z(0)\rangle / L=$ $1-k_{B} T / \sqrt{4 \kappa \sigma_{o}}$, as obtained in Ref. [8].

Equation (10) determines the mean end-to-end distance $\langle z(t)\rangle$ at time $t$ given the full history of the tension $\sigma\left(t^{\prime}\right)$ at all times $0<t^{\prime}<t$. This evolution may be determined uniquely if we identify the physical source of the tension as the Stokes force acting on the bead, Eq. (5). This leads to a set of two equations for the two unknowns $\langle z(t)\rangle$ and $\sigma(t)$. To obtain a single equation, we integrate the Stokes law, Eq. (5), such that

$$
\Sigma(t)=\zeta[\langle z(0)\rangle-\langle z(t)\rangle]
$$

where $\Sigma(t)=\int_{0}^{t} d t^{\prime} \sigma\left(t^{\prime}\right)$. Equation (10) becomes an integral equation for $\Sigma(t)$

$$
\begin{aligned}
\frac{\Sigma(t)}{\zeta L}= & \frac{k_{B} T}{\pi} \int_{\pi / L}^{\pi / a} d k \frac{\left[e^{-2 \Omega_{k}(t)}-1\right]}{\kappa k^{2}+\sigma_{o}} \\
& +\frac{2 k_{B} T}{\pi} \int_{\pi / L}^{\pi / a} d k \Lambda_{k} k^{2} \int_{0}^{t} d t^{\prime} e^{-2\left[\Omega_{k}(t)-\Omega_{k}\left(t^{\prime}\right)\right]},
\end{aligned}
$$

where $\Omega_{k}(t)=\Lambda_{k}\left[\Sigma(t) k^{2}+\kappa t k^{4}\right]$. 
In order to verify the validity of Eq. (12), we consider the (nonphysical) limit where the polymer feels no drag. This may be achieved by formally taking the limit $\Lambda_{k} \rightarrow$ $\infty$. Using $\lim _{\Lambda \rightarrow \infty} \Lambda e^{-\Lambda x}=\delta_{+}(x)$ and performing the integrations in Eq. (12), we obtain the limiting equation of motion:

$$
6 \pi \eta b \frac{d\langle z\rangle}{d t}=-k_{B} T /\left[4 L_{p}(1-\langle z\rangle / L)^{2}\right]=-\sigma_{\mathrm{eq}}(\langle z\rangle) .
$$

Indeed, in this case we expect that the polymer rapidly explores all of its configurations at any given extension, thus passing through quasiequilibrium states as it relaxes. This implies that the forces acting on the bead are the equilibrium polymer force and the Stokes drag force, as described by Eq. (13). While an equation of this type has been previously used to explain the relaxation curve, it failed to quantitatively describe the experimental data [10]. Although reasonable agreement can be obtained using the persistence length as a fitting parameter, the best fitting value is about 3 times larger than the known value, $L_{p} \approx 50 \mathrm{~nm}[10]$.

An alternative form of Eq. (12), which is convenient for numerical analysis, is obtained by writing $\sigma(t)=\sigma_{o}-$ $\delta \sigma(t)$ and integrating by parts the terms involving $t^{\prime}$ explicitly. A further simplification, with little effect on the final result (as verified numerically), is made by replacing $k$ in $\Lambda_{k}$ by $k_{\xi}=1 / \xi$, where $\xi=\sqrt{\kappa / \sigma_{o}}$. Transforming to dimensionless variables $\tilde{k}=k \xi$, $\tilde{t}=$ $t / \tau$, and $\tilde{\Sigma}=\Sigma /\left(\sigma_{o} \tau\right)$, where $\tau=\xi^{2} /\left[2 \Lambda_{k_{\xi}} \sigma_{o}\right]$, and defining $C=3 \ln (\xi / a) b L /\left(\pi \xi L_{p}\right)$, leads to

$$
\begin{aligned}
\tilde{\Sigma}(\tilde{t})= & C \int_{0}^{\tilde{t}} d \tilde{t}^{\prime}\left(1-\frac{d \tilde{\Sigma}\left(\tilde{t}^{\prime}\right)}{d \tilde{t}^{\prime}}\right) \int_{\pi \xi / L}^{\pi \xi / a} d \tilde{k} \frac{\tilde{k}^{2}}{\tilde{k}^{2}+1} \\
& \times e^{-\left(\tilde{t}-\tilde{t}^{\prime}\right) \tilde{k}^{4}-\left[\tilde{\Sigma}(\tilde{t})-\tilde{\Sigma}\left(\tilde{t}^{\prime}\right)\right] \tilde{k}^{2}} .
\end{aligned}
$$

Equation (14) is a nonlinear integrodifferential equation. A straightforward numerical algorithm that uses a single time step has been found to be time consuming. In order to reduce computing time, we divide the total time scale into blocks; each block comprises many integration steps. The time step in each block is constant and doubles from one block to the next. At each time step, Eq. (14) is iteratively solved for $\tilde{\Sigma}(\tilde{t})$. The solution at a given block is based only on half of the data points (i.e., every second point) calculated in the previous block, one quarter of the points calculated in the second previous block, and so on. This algorithm speeds up the computation by about 1.5 orders of magnitude. Once $\tilde{\Sigma}(\tilde{t})$ is found, the extension $z(t)$ is obtained from Eq. (11).

The numerical results were compared to recent measurements on a setup identical to the one described in Refs. $[9,10]$. We use double stranded DNA from the $\lambda$ phage (Promega, $L=16.5 \mu \mathrm{m}$ ). The DNA is attached at one end to a polystyrene bead (Polysciences) and at the other end to the cover slip at the bottom of the sample. This is obtained using a low $p \mathrm{H}$ protocol [9] that allows one to vary $L$. A laser beam (SDL, $\lambda=830 \mathrm{~nm}$ ) is focused through a $100 \times$ objective (Zeiss, 1.3 NA, oil immersion) to give an optical trap. Trapped beads are used to stretch the DNA to extensions that are close to $L$. After turning off the trap, the relaxation is monitored by tracking the bead at video rate, 25 frames/s, and with low spatial resolution, $0.3 \mu \mathrm{m}$. Experiments were performed with different bead sizes, $b=0.5,1$, and $1.4 \mu \mathrm{m}$.

In Figs. 1-3, we compare the predictions of our theory to the experimental data. In the numerical simulations, we used the viscosity of water at room temperature $\eta=$ $0.89 \mathrm{mPs}$, DNA persistence length $L_{p}=50 \mathrm{~nm}$, and DNA thickness $a=2 \mathrm{~nm}$. There are no free parameters. The initial extension of the DNA molecule known from
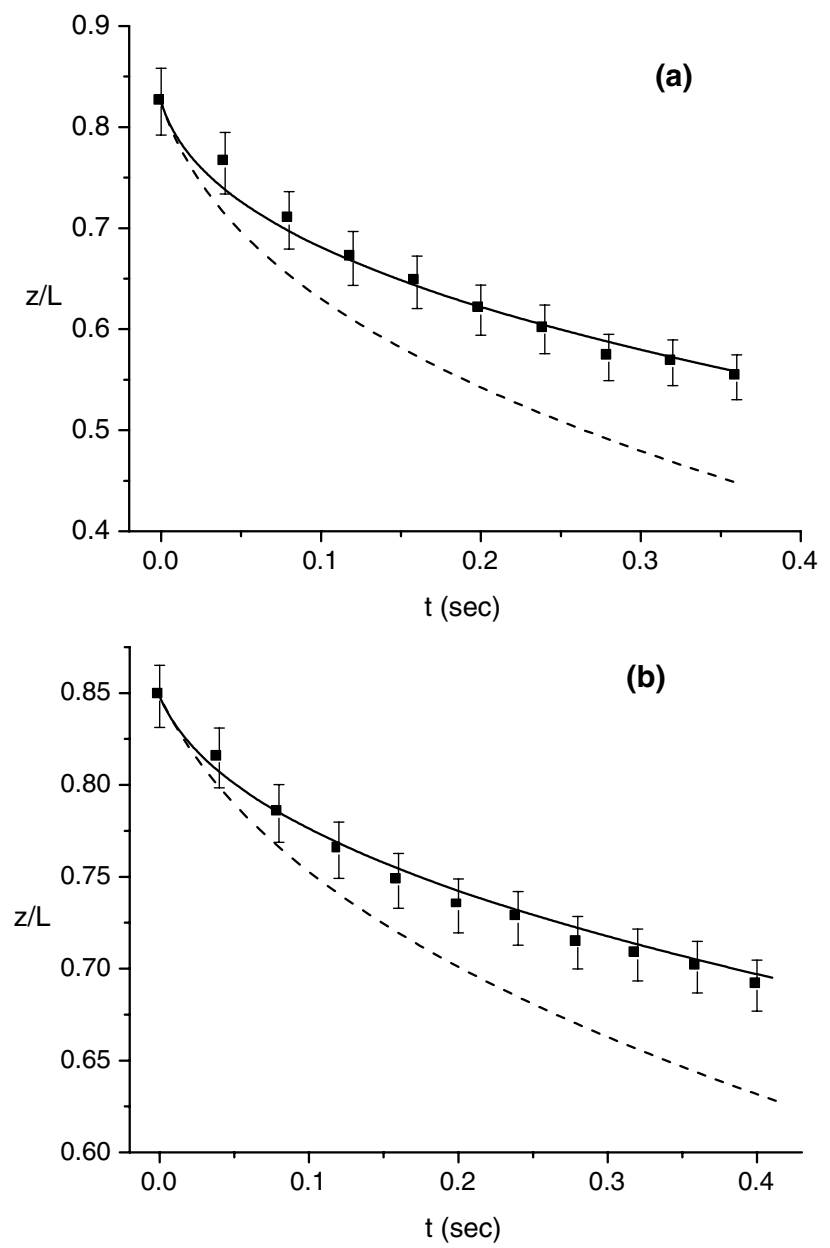

FIG. 1. DNA extension plotted vs time. (a) DNA length $L=$ $14.5 \mu \mathrm{m}$, bead radius $b=0.5 \mu \mathrm{m}$, and initial extension $z(0)=$ $0.825 L$; (b) $L=20.1 \mu \mathrm{m}, b=1.4 \mu \mathrm{m}$, and $z(0)=0.848 L$. The solution of Eqs. (11) and (14) (solid line) and the experimental data points are shown. The computational error in the solid line is about $4 \%$ at the longest times shown and diminishes at shorter times. For comparison, the solution of Eq. (13) (dashed line) is also shown. 


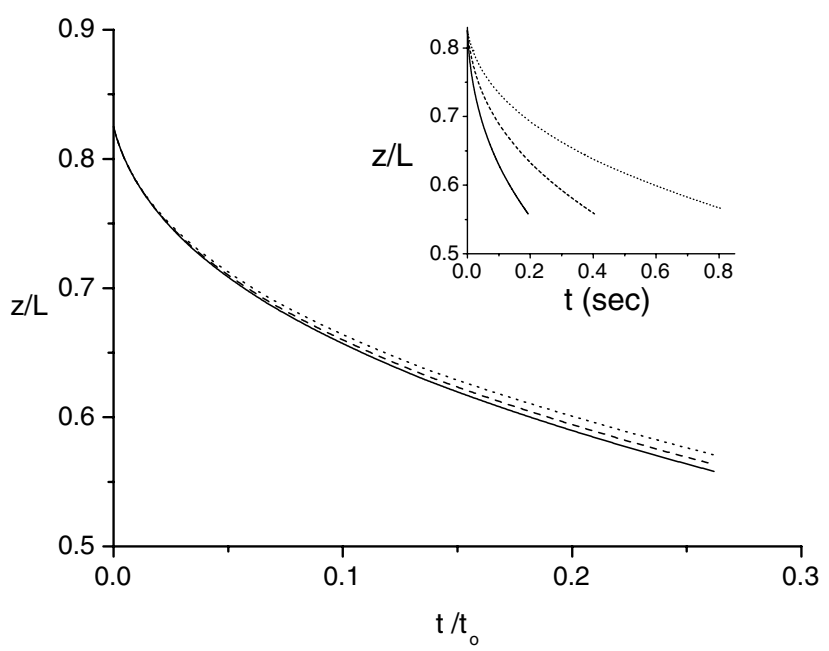

FIG. 2. Theoretical extension, computed from Eqs. (11) and (14), vs the scaled time $t / t_{o}$, where $t_{o}=6 \pi \eta L_{p} L b / k_{B} T$, for three different bead radii: $b=0.25 \mu \mathrm{m}$ (full line), $b=0.5 \mu \mathrm{m}$ (dashed line), and $b=1 \mu \mathrm{m}$ (dotted line). $L=14.5 \mu \mathrm{m}$ and $z(0) / L=0.825$. Inset: Same as above but against the time (unscaled).

the experiment determines the initial tension used in the numerical solution, $\sigma_{o}=k_{B} T /\left\{4 L_{p}[1-z(0) / L]^{2}\right\}$. In Figs. 1(a) and 1(b), we plot the relative extension $z / L$ against time $t$ for two systems differing in chain length, bead size, and initial conditions. Good agreement between theory and experiment is found. For comparison, we also show the solution of Eq. (13) (dashed line) which fails to account for the experimental data. Figure 2 shows theoretical predictions for a system with three different bead sizes, with the time rescaled to $t / t_{o}$, where $t_{o}=$ $6 \pi \eta L_{p} L b / k_{B} T$, while in the inset we plot the results against time. We find that the collapse of the three theoretical curves is nearly perfect, signifying a weak breakdown of scaling. A nearly perfect scaling is also found when we vary the chain length $L$. Note that this scaling cannot be inferred directly from Eq. (14), although it is obeyed by the $b \rightarrow \infty$ limiting Eq. (13). In Fig. 3, we demonstrate a similar scaling behavior in the experimental data.

In this Letter, we have developed a first principle approach to dynamics of strongly stretched semiflexible chains. This approach employs a time-dependent tension that is uniquely determined by a supplementary physical constraint. By comparing to experiments, we have shown that our theory works well with no free parameters. Our approach may be extended to other problems, e.g., the nonlinear viscoelasticity of actin networks and the dynamics of DNA-protein complexes [13].

We thank R. Baer, D. Chatenay, M. Elbaum, E. Frey, E. Goshen, T. Liverpool, F. MacKintosh, A. Maggs, and D. Morse for useful discussions. This work was supported in part by the National Science Foundation under Grant

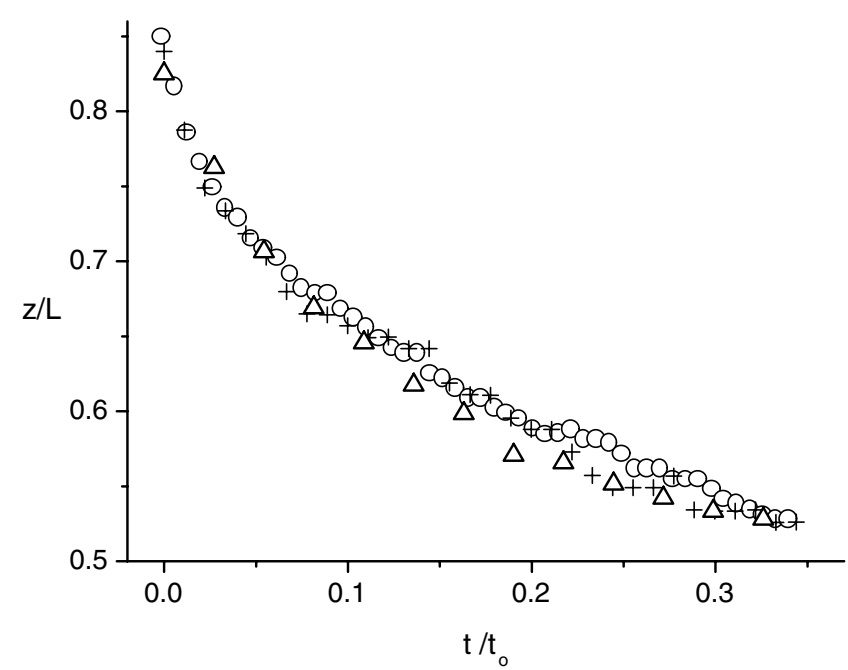

FIG. 3. Experimental results for DNA relaxation. The time axis is divided by $t_{o}$. Circles are for $b=1.4 \mu \mathrm{m}$ [same system as for Fig. 1(b)], plusses are for $b=0.5 \mu \mathrm{m}$ [same system as for Fig. 1(a)], and triangles are for a system with $b=1 \mu \mathrm{m}$, $z(0) / L=0.84$, and $L=17.7 \mu \mathrm{m}$.

No. PHY99-07949, and by the Israel Academy of Science and Humanities (Grant No. 263/00).

*Author to whom correspondence should be addressed. Electronic address: rgranek@bgumail.bgu.ac.il

[1] F. Amblard et al., Phys. Rev. Lett. 77, 4470 (1996); L. Le Goff et al., Phys. Rev. Lett. 89, 258101 (2002); A. Caspi et al., Phys. Rev. E 66, 011916 (2002).

[2] F. Gittes and F. C. MacKintosh, Phys. Rev. E 58, R1241 (1998); E. Farge and A. C. Maggs, Macromolecules 26, 5041 (1993); K. Kroy and E. Frey, Phys. Rev. E 55, 3092 (1997.

[3] V. Shankar et al., J. Rheol. 46, 1111 (2002); T. B. Liverpool and A.C. Maggs, Macromolecules 34, 6064 (2001); R. Everaers et al., Phys. Rev. Lett. 82, 3717 (1999).

[4] R. Granek, J. Phys. II (Paris) 7, 1761 (1997).

[5] T. T. Perkins et al., Science 264, 822 (1994); 268, 83 (1995).

[6] F. Brochard-Wyart et al., Europhys. Lett. 26, 511 (1994); S. Manneville et al., Europhys. Lett. 36, 413 (1996).

[7] S. B. Smith et al., Science 258, 1122 (1992); 271, 795 (1996); P. Cluzel et al., Science 221, 792 (1996); J. C. Meiners and S. R. Quake, Phys. Rev. Lett. 84, 5014 (2000); T. R. Strick et al., Science 271, 1835 (1996).

[8] J. Marko and E. Siggia, Macromolecules 28, 8759 (1995).

[9] G. V. Shivashankar et al., Proc. Natl. Acad. Sci. U.S.A. 96, 7916 (1999).

[10] M. Feingold, Physica (Amsterdam) 9E, 616 (2001).

[11] U. Seifert et al., Phys. Rev. Lett. 77, 5389 (1996).

[12] F. Brochard-Wyart et al., Europhys. Lett. 47, 171 (1999).

[13] H. Salman et al., Biophys. J. 80, 783 (2001). 